\title{
Precompetition anxiety in women volleyball players: a test of ZOF theory in a team sport
}

\author{
John S. Raglin PhD and Mary J. Morris MS \\ Indiana University, Department of Kinesiology, Bloomington, Indiana USA
}

\begin{abstract}
Consistency in psychological factors is widely regarded to be important for successful performance in team sports, but the Zone of Optimal Function (ZOF) theory contends that athletes should exhibit considerable variability in the level of anxiety that will optimize performance. In an attempt to determine if tenets of ZOF theory held for athletes in a team sport, anxiety was measured using Spielberger's state-trait anxiety inventory (STAI) at the baseline and before easy and difficult competitions in nine members of a collegiate women's volleyball team. The ability to predict precompetition anxiety was assessed by having the athletes complete the STAI both 3 weeks and 2 days before each match according to how they thought they would feel $1 \mathrm{~h}$ before competition. Each athlete also completed the STAI on the basis of how she recalled feeling before her best competition. Four anxiety units were added and subtracted from this value to establish the ZOF of each player. Actual precompetition anxiety was assessed $1 \mathrm{~h}$ before each match. In accordance with ZOF theory, considerable variability was found in the range of optimal anxiety, and $55.5 \%$ of the team members reported performing best at either low or high levels of anxiety. The prediction of precompetition anxiety made 2 days before competition was significantly correlated to actual anxiety for the difficult match $(r=0.69, P<$ $0.05)$ but not the easy match $(r=0.21, P>0.05)$. Predictions made 3 weeks before competition were not significant $(P>0.05)$. More $(P<0.05)$ of the players possessed anxiety levels within the ZOF for the difficult match compared with the easy match $(77.7 \%$ versus $22.2 \%$ ). In summary, athletes in the team sport of volleyball exhibit considerable variation in optimal precompetition anxiety in accordance with ZOF theory. As posited by ZOF theory, the athletes were able to predict anxiety before a difficult match accurately, and were more likely to have anxiety levels within ZOF.
\end{abstract}

Keywords: Anxiety, competition, ZOF theory, performance

In the field of sport psychology the predominating explanation for the influence of anxiety on sport performance has been the inverted- $U$ hypothesis ${ }^{1-6}$. Although originally intended to describe the effects of physiological arousal, the inverted- $U$ hypothesis has been interpreted to posit that when anxiety lies within a moderate range, performance will be

Address for correspondence: John S. Raglin PhD, HPER Building Room 112, Department of Kinesiology, Indiana University,

Bloomington, Indiana 47405, USA

(C) 1994 Butterworth-Heinemann Ltd

0306-3674/94/010047-05 maximized. As anxiety either falls below or exceeds moderate levels, performance rapidly worsens. Recent conceptualizations of the inverted- $U$ hypothesis contend that athletic experience and the motor demands of the sport task dictate where the optimal midrange lies on the anxiety continuum ${ }^{1,2,5}$.

Despite the central role of the inverted- $U$ hypothesis in sport psychology, it has come under criticism in a number of recent reviews. Problems noted include a lack of supportive evidence ${ }^{3,4,6,7}$, and the tendency of the hypothesis to minimize the importance of individual differences in the relationship between anxiety and performance ${ }^{4}$. An alternative theory that explicitly incorporates the concept of individual differences has been developed by Hanin $^{8,9}$. Hanin's Zone of Optimal Function (ZOF) theory - recently referred to as Individual Zones of Optimal Functioning theory - indicates that each athlete possesses an optimal anxiety range for sport performance. This zone of optimal function may be low, moderate, or high, depending on the individual, and it is not systematically influenced by either the motor demands of the activity or athletic experience. Unlike the inverted- $U$ hypothesis, ZOF theory indicates that among athletes of similar skill competing in a given sport, there will be no common range of optimal anxiety; many will benefit from low or high anxiety levels.

An athlete's ZOF can be established directly by assessing anxiety before competition until a personal best performance results. However, because this procedure requires considerable time and effort, Hanin $^{8,9}$ has developed an alternative where ZOF is determined by having athletes complete the anxiety questionnaire according to how they recall feeling before their own best competition. These recalled optimal anxiety values have been shown to correlate highly ( $r=0.60$ to 0.80 ) with anxiety levels actually experienced before competition 9,10 . Research by Hanin ${ }^{9}$ and others ${ }^{10-14}$ indicates that athletes are also able to predict their own level of precompetition anxiety up to several days before the actual competition. Correlations between predicted precompetition anxiety and actual values range from $r=0.60$ to $r=$ 0.80 , with higher coefficients occurring when the competition is more difficult.

However, much of the evidence in support of ZOF theory comes from studies of individual sports ${ }^{9,13,14}$, and there has been a general lack of ZOF research in team sports. Because psychological factors such as 
cohesiveness $^{15}$ and homogeneity of social variables ${ }^{16}$ are regarded to be especially important for success in team sports, it is feasible that the high degree of variability in precompetition anxiety posited by ZOF theory would be a liability for team sport athletes. If this were true, then the inverted- $U$ hypothesis may yet hold in such cases.

In an effort to determine if ZOF theory is applicable to a team sport the following study was conducted. Anxiety was measured at baseline and before an easy and difficult competition in members of a college varsity volleyball team. Based upon the assumptions of ZOF theory, it was hypothesized that the team members would exhibit significant variability in actual precompetition anxiety and ZOF levels. Based upon previous research ${ }^{13}$, it was also hypothesized that successful performers would be more likely to have precompetition anxiety values within $Z O F$. The ability of these athletes to predict precompetition anxiety and to recall past precompetition anxiety was also assessed in an effort further to validate suppositions of ZOF theory.

\section{Subjects and methods}

A general overview of the study was presented to members of a university women's varsity volleyball team. Those who agreed to participate then completed an informed consent document before further involvement. To preserve the confidentiality of the subjects, the coach agreed not to be informed of who participated in the study, and was not provided with individual results.

Before the beginning of the competitive season, the subjects completed the state-trait anxiety inventory (STAI) ${ }^{17}$. The STAI consists of two, 20-item scales that measure state and trait anxiety. The athletes also completed a version of the STAI where each subject responded to the items according to how you recall feeling just prior to your best performance'. According to procedures outlined by Hanin ${ }^{9}$, four anxiety units were added and subtracted from this recalled optimal value to establish the ZOF of each athlete.

Each athlete also completed the STAI according to how she anticipated feeling before the upcoming competition. This questionnaire was administered 19 days before the easy match, 22 days before the difficult match, and again 2 days before both matches. Actual precompetition anxiety was assessed $1 \mathrm{~h}$ before both matches with the STAI using the standard instructional set. Finally, 3 months following the difficult match the athletes completed the STAI according to how anxious they remembered feeling before the difficult match. This value was used to evaluate the ability of athletes to recall precompetition anxiety associated with past competitions.

In an effort to obtain a dynamic measure of individual success, the total number of volleys (i.e. play resulting in a side out or point) for which each player was on court was counted in each match. This value was divided by the total number of volleys for the match and then multiplied by 100 to yield the percentage of total playing time completed by each player. To create groups of successful and unsuccessful athletes, a median split was formed using percentage of total playing time. It was assumed that team members who performed more successfully would get significantly greater playing time compared with those who did more poorly.

One easy and one difficult competition was selected by the coach on the basis of past records and other available information. The coach's judgment turned out to be accurate; the team won the easy match in straight games (three to zero), whereas four games were needed for the team to win the difficult match. Athletes in the successful group participated in all volleys in both matches, whereas athletes in the unsuccessful group participated in $39.3 \%$ of the volleys in the easy match and $26.0 \%$ in the difficult match.

\section{Results}

\section{Descriptive findings}

Complete data were available for nine (age range: 18-22 years) subjects. Means and standard deviations of the baseline, predicted, and actual precompetition anxiety data are presented for both matches in Table 1. A one-way repeated measures analysis of variance was carried out for baseline, predicted, and actual precompetition anxiety values in the easy and difficult match. None of the actual or predicted values differed $(P>0.05)$ from the baseline with one exception. The mean value of predicted precompetition anxiety made 2 days before the easy match was elevated $(P<0.05)$ above both baseline and actual anxiety for the easy match. Although actual precompetition anxiety was not significantly $(P>0.05)$ elevated above baseline for either match, when the data were examined on an individual basis, some of the athletes were revealed to possess elevated precompetition anxiety. For each match $22.2 \%$ (two of nine) of the athletes possessed precompetition anxiety levels that were at least one standard deviation above the mean of the entire team.

Table 1. Baseline, predicted and actual precompetition anxiety

\begin{tabular}{|c|c|c|c|c|c|c|c|}
\hline \multicolumn{2}{|c|}{ Baseline } & \multicolumn{3}{|c|}{ Easy match } & \multicolumn{3}{|c|}{ Difficult match } \\
\hline State & Trait & 19-Day predicted & 2-Day predicted & Actual & 22-Day predicted & 2-Day predicted & Actual \\
\hline $41.4(10.8)$ & $39.7(13.1)$ & $38.0(4.0)$ & $46.4^{*}(8.9)$ & $39.7(6.3)$ & $41.2(5.3)$ & $42.6(5.8)$ & $39.7(7.8)$ \\
\hline
\end{tabular}

Values are mean (s.d.); ${ }^{*} P<0.05$ above baseline state anxiety; analysis of variance and post hoc Newman-Kuels 


\section{Variability in optimal anxiety}

The variability in optimal anxiety of the athletes was examined by using previously established categories ${ }^{10}$ based on anxiety ranges. The anxiety ranges are as follows: low, one-half standard deviation or more below the published norm; moderate, within one-half standard deviation above or below the norm; and high, one-half standard deviation or greater than the norm. When the optimal anxiety values were categorized using these ranges it was revealed that $22.2 \%$ (two of nine) of the athletes had optimal anxiety levels that fell in the low anxiety category, $44.4 \%$ (four of nine) in the moderate category, and $33.3 \%$ (three of nine) in the high category.

\section{ZOF and match difficulty}

To determine if the athletes were more likely to have achieved ZOF in the difficult match, the number of team members that were within their own ZOF was compared between matches. The resulting percentages were contrasted with a Z-score transformation test of proportions ${ }^{18}$. Precompetition anxiety values were within the ZOF for $22.2 \%$ (two of nine) of the athletes in the easy match. In the difficult match $77.7 \%$ (seven of nine) of the team members had values within the $Z O F$, a significantly $(Z=2.41, P<$ 0.01 ) greater percentage compared with the easy match.

\section{ZOF and athletic success}

On the basis of previous $\mathrm{ZOF}$ research ${ }^{13}$ it was anticipated that successful players would be more likely to be within their own ZOF than the less successful team members. For each match the percentage of athletes with precompetition anxiety levels within their own ZOF was determined for the successful and unsuccessful groups. The differences between these percentages were contrasted by using $Z$-score tests of proportions ${ }^{18}$, and no significant differences between the groups were found for either the easy or difficult match $(P>0.05)$.

\section{Predicted and actual precompetition anxiety}

Pearson correlation coefficients were derived to determine the capability of the athletes to predict precompetition anxiety at different intervals before the matches, and the coefficients for both matches are presented in Table 2. For the difficult match, a

Table 2. Pearson correlation coefficients between predicted and actual precompetition anxiety

\begin{tabular}{ccccc}
\hline \multicolumn{2}{c}{ Easy match } & & \multicolumn{2}{c}{ Difficult match } \\
\cline { 4 - 5 } $\begin{array}{c}\text { 19-Day } \\
\text { prediction }\end{array}$ & $\begin{array}{c}2-\text { Day } \\
\text { prediction }\end{array}$ & & $\begin{array}{c}22-\text { Day } \\
\text { prediction }\end{array}$ & $\begin{array}{c}\text { 2-Day } \\
\text { prediction }\end{array}$ \\
\hline 0.59 & 0.21 & & 0.41 & $0.69 *$ \\
\hline
\end{tabular}

$* P<0.05$

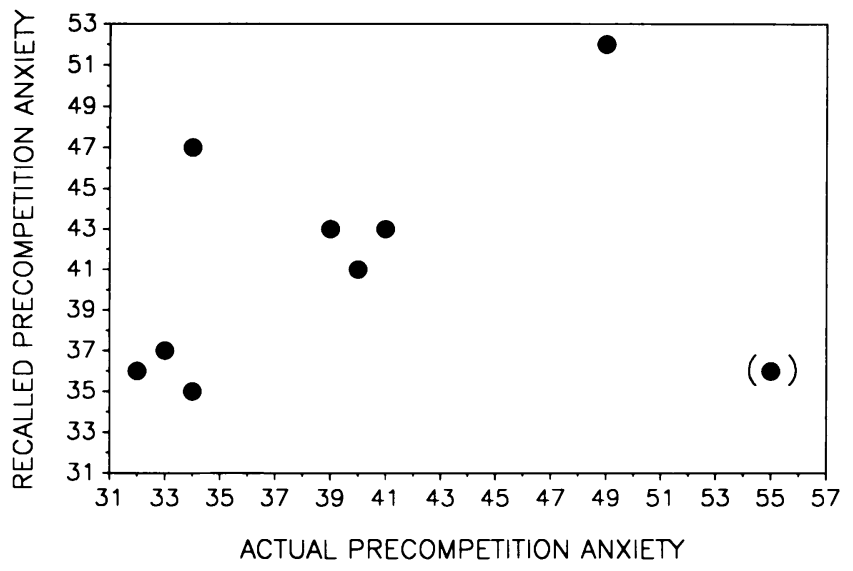

Figure 1. Recalled and actual precompetition anxiety (STAI units) values for a difficult match

significant $(P<0.05)$ correlation of 0.69 was found between actual precompetition anxiety and predictions made 2 days earlier, whereas the correlation made 22 days earlier was not significant $(r=0.41, P>$ 0.05 ). Correlations between predicted and actual precompetition anxiety were not significant at either time interval for the easy match.

\section{Accuracy of recalling past precompetition anxiety}

Recalled precompetition anxiety values for the difficult match were obtained in the subjects 92 days after the actual match, and then compared via correlational analysis. A nonsignificant $(P>0.05)$ correlation of 0.26 was found between the actual and recalled values and the findings are presented in Figure 1. Inspection of the data revealed an outlier, indicated in Figure 1 by parentheses. When the values for this subject were removed from the analysis, a significant $(P<0.05)$ correlation of 0.75 was obtained. Coefficients were also calculated after the data of each of the other subjects were removed singly from the analysis, but in no case did this result in a significant correlation. Hence, the scores of one subject resulted in the lack of significance of the correlation based on data from all nine athletes.

\section{Discussion}

The findings of the present study indicate that many of the tenets of ZOF theory are applicable to team sports. Although mean precompetition anxiety was not elevated above the baseline for either match, $22 \%$ of the team members reported anxiety values that were significantly elevated above the baseline. Similarly, Raglin and Turner ${ }^{10}$ found that $32 \%$ of a sample of track and field athletes possessed high precompetition anxiety levels, although mean precompetition anxiety of the entire sample was not elevated above the baseline. The volleyball players also displayed considerable variability in optimal precompetition anxiety. Over $55 \%$ of the sample reported that best performance occurred when anxiety was either low or high. This finding contradicts a tenet of the inverted- $U$ hypothesis that posits optimal anxiety should lie within the moderate 
range. One-third of the players had optimal anxiety levels in the high range, and this percentage is similar to those reported for athletes in individual sports ${ }^{12,19}$. The results of this study indicate that athletes in team sports such as volleyball display a range of variability in actual and optimal anxiety comparable to that found among athletes in individual sports. Although the external validity of findings yielded from a single team consisting of a small number of athletes is necessarily limited, the addition of subjects from other teams may well have presented greater threats to validity. Pooling data from additional teams could result in uncontrollable differences across subjects in factors such as athletic skill, coaching practices or competitive setting. Future research in this area will need to address these challenges, as well as examine the viability of ZOF theory in additional team sports.

Consistent with previous ZOF research ${ }^{20}$, more of the team members were within the $\mathrm{ZOF}$ for the difficult match than the easy competition. This finding supports the view that the achievement of ZOF may be crucial for adequate performance only in difficult competitions ${ }^{13}$. However, unlike previous research $^{13}$, the successful performers were not more likely to achieve ZOF than their unsuccessful teammates. Although this finding suggests that ZOF is not important for successful performance, it is also possible these results reflect weaknesses in the performance measure used. Although the use of playing time percentages provides advantages over static measures of performance such as playing status (i.e. starter or nonstarter ${ }^{15}$, relevant factors such as health or training status were not considered. It also should be recognized that the appraisal of athletic success in team sports is problematic as the overall success of the team may be independent of the performance of any single athlete. Future ZOF research with team sports should utilize multidimensional measures of performance which not only include objective game statistics, but also information on pertinent factors such as health status.

Predicted precompetition anxiety was significantly correlated to actual anxiety $(P<0.05)$ only for the difficult match, and the accuracy in predicting precompetition anxiety improved as the difficult match approached. Significant correlations were not found for the easy match, and the mean value of predicted anxiety made 2 days before the easy meet was significantly higher than actual precompetition anxiety. Previous research ${ }^{12,13}$ has also found that female athletes are inaccurate in predicting precompetition anxiety in easy meets $(r=0.27, r=0.39)$ but are accurate $(r=0.95, r=0.77)$ when meets are difficult. Moreover, in these studies ${ }^{12,13}$ the values for predicted precompetition anxiety for easy meets were significantly higher than the actual anxiety values, and this also occurred in the present study. These findings, in tandem with other research ${ }^{10}$, suggest that female athletes are accurate in predicting anxiety for difficult competitions, but tend to overestimate their own levels of precompetition anxiety for easy competitions. This has not been observed in male athletes ${ }^{10,11}$, and research is needed to determine if factors such as athletic experience or socialization contribute to these differences.
The findings for the 3-month recall of precompetition anxiety of the difficult match provided some support for Hanin's ${ }^{8,9}$ contention that athletes can accurately recall past precompetition anxiety. The correlation between recalled and actual anxiety was $r=0.26$ for the entire sample, but after dropping the scores of an obvious outlier the correlation increased to $r=0.75(P<0.05)$; a value similar to coefficients found by Hanin ${ }^{9}$ and others ${ }^{14,20}$. Over $87 \%$ (seven of eight) of the volleyball players had recalled anxiety values within their ZOF (i.e. within four anxiety units from optimal anxiety either way), and this level of accuracy is considerably higher than the $27 \%$ found by Imlay et al..$^{20}$ in a 3-month recall test with track and field athletes. The present findings indicate that most athletes should be accurate in recalling their own optimal anxiety level. It is not clear why some individuals fail in this task, and further research will be needed to determine what factors are responsible. However, the use of a 3-month or 4-month precompetition anxiety recall could be used to identify athletes likely to be inaccurate in recalling optimal precompetition anxiety. For such individuals, it may be advisable to determine their ZOF directly by assessing precompetition anxiety before a personal best performance.

In summary, it was found that members of a university volleyball team exhibited considerable variability in both actual and optimal precompetition anxiety in accordance with the tenets of ZOF theory. These athletes were accurate in predicting their own levels of precompetition anxiety before a difficult match, and the majority could accurately recall their own level of precompetition anxiety in a match that took place 3 months earlier. The considerable variation found in actual and optimal precompetition anxiety levels goes against the belief that consistency in anxiety $^{1}$ or other psychological factors ${ }^{15,16}$ is important for success in team sport athletes.

\section{References}

1 Cox RH. Arousal in sport. Sport Psychology: Concepts and Applications. Dubuque, Indiana, USA: William C. Brown, 1990; 2: 87-115.

2 LeUnes AD, Nation JR. Anxiety, arousal, and intervention. In: Sport Psychology: an Introduction. Chicago, Illinois, USA: Nelson-Hall, 1989: 95-120.

3 Morgan WP, Ellickson KA. Health, anxiety, and physical exercise. In: Hackfort D, Spielberger CD, eds. Anxiety in Sports: An International Perspective. Washington, DC, USA: Hemisphere Publishing, 1989: 165-82.

4 Neiss R. Reconceptualizing arousal: psychobiological states in motor performance. Psychol Bull 1988; 103: 345-66.

5 Oxendine JB. Emotional arousal and motor performance. Quest 1970; 13: 23-32.

6 Raglin JS. Anxiety and sport performance. In: Holloszy JO, ed. Exercise and Sport Sciences Reviews. Baltimore, Maryland, USA: Williams and Wilkins 1992; 20: 243-74.

7 Fazey J, Hardy L. The Inverted- $U$ Hypothesis: $A$ Catastrophe for Sport Psychology? Leeds, UK: White Line Press, 1988.

8 Hanin YL. A study of anxiety in sports. In: Straub WF, ed. Sport Psychology: An Analysis of Athletic Behavior. Ithaca, New York, USA: Mouvement Publications, 1978: 236-49.

9 Hanin YL. State-trait research on sports in the USSR. In: Spielberger CD, Diaz-Guerrero R., eds. Cross-Cultural Anxiety. USA: Washington, DC, Hemisphere Publishing, 1986; 3: 45-64. 
Raglin JS, Turner PE. Predicted, actual and optimal precompetition anxiety in adolescent track and field athletes. Scand J Med Sci Sports 1992; 2: 148-52

11 Raglin JS, Morgan WP. Predicted and actual levels of pre-competition state anxiety in swimmers. J Swimming Res 1988; 4: 5-8.

12 Raglin JS, Wise KJ, Morgan WP. Predicted and actual pre-competition anxiety in high school girl swimmers. Swimming Res 1990; 2: 5-8.

13 Raglin JS, Morgan WP, Wise KJ. Pre-competition anxiety and performance in female high school girl swimmers: a test of optimal function theory. Int I Sports Med 1990; 11: 171-5.

14 Raglin JS, Turner PE. Anxiety and performance in track and field athletes: a comparison of the inverted- $U$ hypothesis with zone of optimal function theory. Person Individ Diff. New York, USA: W. C. Brown, 1993; 14: 163-71.

15 Spink KS. Group cohesion and starting status in successful and less successful elite volleyball teams. I Sports Sci 1992; 10: 379-88.

16 Cox RH. Team Cohesion. Sport Psychology: Concepts and Applications. Dubuque, Indiana, USA: William C. Brown, 1990; 2: 335-69.

17 Spielberger CD, Gorsuch RL, Lushene RE et al. Manual for the State-Trait Anxiety Inventory STAI (Form Y). USA: Consulting Psychologists Press, 1983.

18 Ferguson GA. Statistical Analysis in Psychology and Education. New York, USA: McGraw Hill, 1966: 177-81.

19 Morgan WP, O'Connor PJ, Sparling PB, Pate RR. Psychological characterization of the elite female distance runner. Int Sports Med 1987; (Suppl.) 8: 124-31.

20 Imlay GJ, Carda RD, Stanbrough ME, O'Connor PJ. Anxiety and athletic performance: a test of optimal function theory. Med Sci Sports Exerc 1992; (Suppl.) 24: S150.

\title{
STOKE SPORTS SEMINARS
}

\section{Advanced Sports Medicine Course in Two Modules for Doctors and Physiotherapists}

\author{
Stakis Grand Hotel, Stoke-on-Trent
}

Module 1: $\quad$ The Injured Knee

Date:

April 30th - May 1st, 1994

Module 2: $\quad$ Cruciate Ligament Injury

Date: $\quad$ May 14th - 15th, 1994

Course Fee: $£ 250$ for each Module. Inclusive of all meals and overnight accommodation at the course venue.

\section{P.G.E.A. Approved.}

Application forms and programmes available from:

Stoke Sports Seminars

Harpfields Sports Medicine Clinic

1 Longfield Road

Hartshill

Stoke-on-Trent

ST4 6QN 